\title{
A Switch from Cycloheximide-Resistant Consolidated Memory to Cycloheximide-Sensitive Reconsolidation and Extinction in Drosophila
}

\author{
Fabrice Lagasse, ${ }^{1}$ Jean-Marc Devaud, ${ }^{2}$ and Frederic Mery ${ }^{1}$ \\ ${ }^{1}$ Laboratoire Évolution, Génomes et Spéciation, Centre National de la Recherche Scientifique, Unité Propre de Recherche 9034, 91198 Gif-sur-Yvette Cedex, \\ France, and ${ }^{2}$ Centre de Recherches sur la Cognition Animale, Centre National de la Recherche Scientifique, Unité Mixte de Recherche 5169, Université Paul \\ Sabatier, 31062 Toulouse, France
}

It is generally accepted that, after learning, memories stabilize over time and integrate into long-term memory (LTM) through the process of consolidation, which depends on de novo protein synthesis. Besides, studies on several species have shown that reactivation of already stabilized LTM can either make this memory labile and then restabilize it (a process called reconsolidation) or inhibit it (extinction). However, the identity of both processes and their interactions with consolidation are still under debate. Regarding memory stabilization, Drosophila offers a striking exception since, in this species, LTM is not the sole stable form of memory. Under specific learning conditions, anesthesia-resistant memory (ARM) can be formed through processes yet unknown but that are resistant to cycloheximide, a classical protein synthesis inhibitor that impairs LTM. Here, we took advantage of this dichotomy to ask whether both ARM and LTM could be extinguished and/or reconsolidated. We also studied whether two forms of memory extinction and reconsolidation exist in flies, as for memory stabilization. We show that either reconsolidation or extinction can be induced after olfactory conditioning in Drosophila, depending on the number of reactivations as in other species. Furthermore, regarding the effect of cycloheximide, the ARM/LTM dichotomy for stabilization does not apply to extinction and reconsolidation. Blocking protein synthesis interfered with both processes regardless of whether initial stabilization was sensitive (LTM) or not (ARM) to cycloheximide. These results thus show that Drosophila is a useful model to tackle these questions and that reconsolidation is not necessarily a mere repetition of consolidation.

Key words: consolidation; reconsolidation; extinction; associative conditioning; protein synthesis; invertebrate

\section{Introduction}

Many studies have shown that the maintenance of memories requires consolidation, a crucial phase which allows the learned information to become resistant to interfering treatments (Duncan, 1949; Dudai and Morris, 2000; Dudai and Eisenberg, 2004). Until recently, it was considered that, after a single consolidation phase, memories remained resistant to interference (McGaugh, 2000). However, several studies have challenged this view. After consolidation of a memory of an association between a conditioned stimulus (CS) and an unconditioned stimulus (US), further presentations of the CS alone (reactivation) can destabilize it. Once made labile, the initial memory trace (CS-US) can be stabilized again through reconsolidation (Misanin et al., 1968; Mactutus et al., 1979; Nader et al., 2000; Sara, 2000; Dudai, 2006), so that the behavioral response to the CS is maintained. Alternatively, reactivation can lead to extinction, a decrease of the re-

Received Aug. 11, 2008; revised Dec. 12, 2008; accepted Dec. 31, 2008.

This work was supported by an Action Thématique et Incitative sur Programme grant from the Life Sciences Division of the Centre National de la Recherche Scientifique to F.M. We thank J. Burns for useful comments on this manuscript.

Correspondence should be addressed to Frederic Mery, Laboratoire Évolution, Génomes et Spéciation, Avenue de la Terrasse, Bâtiment 13,91198 Gif-sur-Yvette Cedex, France. E-mail: Frederic.mery@legs.cnrs-gif.fr. DOI:10.1523/JNEUROSCI.3789-08.2009

Copyright $\odot 2009$ Society for Neuroscience $\quad$ 0270-6474/09/292225-06\$15.00/0 sponse resulting from the consolidation of a new memory of the association CS-no US (Suzuki et al., 2004).

Reconsolidation and extinction thus lead to opposite behavioral outcomes. Whether one or the other process is triggered depends on several factors (Alberini et al., 2006). In particular, as shown in rats by Suzuki et al. (2004), the strength and age of the original memory is important, as is the strength of the reactivation phase (number/duration of reactivation trials), such that younger and weaker memories are more easily reconsolidated. In species usually studied, consolidation, extinction, and reconsolidation are processes of memory stabilization, and require de novo protein synthesis. However, what their underlying mechanisms are is still under debate. In particular, the question of the similarity between consolidation and reconsolidation remains disputed (Alberini, 2005; Nader et al., 2005; Alberini et al., 2006; Eisenhardt and Menzel, 2007; Moore and Roche, 2007; Stollhoff et al., 2008).

To clarify the relationships between these processes, we studied some of their properties in Drosophila melanogaster, which provides genetic tools that should ultimately help to define aspects of their cellular and molecular bases. We used the common learning paradigm of classical (Pavlovian) olfactory conditioning, in which the CS is an odorant and the US a shock, whether electric (Tully et al., 1994) or mechanical (Mery and Kawecki, 
2005). A mutant-based genetic dissection of memory has distinguished four kinds of memory using electric shock as US (Tully et al., 1994). Beside the labile short-term memory and middle-term memory are two forms of stabilized memory: a cycloheximide (CXM)-insensitive memory [anesthesia-resistant (ARM)] and a CXM-sensitive (protein synthesis-dependent) long-term memory (LTM). They can be induced experimentally through multiple CS-US presentations during conditioning: ARM is produced when no delay separates successive presentations of the CS paired with the US (massed protocol) while a sufficient delay (spaced protocol) produces both ARM and LTM (Tully et al., 1994). This study aimed to analyze the effect of reactivation on these two forms of memory, by evaluating whether extinction and/or reconsolidation could be induced by different numbers of reactivation trials, and whether the nature of the initial memory had an impact on these processes.

\section{Materials and Methods}

Fly stock and maintenance. Adult wild-type Drosophila melanogaster flies collected in the center of France (Chavroches) in 2006 and maintained in the lab on standard food were used for all experiments.

Conditioning. We used a classical aversive olfactory conditioning assay (Mery and Kawecki, 2005). Flies were conditioned to associate one of two odors (3-octanol or 4-methylcyclohexanol, Sigma) with a mechanical shock. Conditioning was performed on groups of 50 adult flies (sexes mixed) aged 3-5 d from eclosion. These flies were isolated from culture bottles $24 \mathrm{~h}$ before conditioning, placed in small vials containing a wet filter paper, and kept at $22^{\circ} \mathrm{C}$ until conditioning. The conditioning procedure consisted of five training sessions separated by 20 min intervals (spaced protocol) or immediately after one another (massed protocol). In each training session, flies were first exposed for $1 \mathrm{~min}$ to the CS+ odor (3-octanol or 4-methylcyclohexanol) simultaneously with mechanical shock (2000 rpm vibration pulses of $1 \mathrm{~s}$ duration, delivered every $5 \mathrm{~s}$ by a test tube shaker). This period was followed by a $1 \mathrm{~min}$ rest period, during which flies received humid air flow (no odor) and no shocks. Then, for $1 \mathrm{~min}$, the other odorant was delivered, without shock. The training session ended with a second rest period of $1 \mathrm{~min}$. Half of the flies were conditioned with 3-octanol as CS+ and the other half with 4-methylcyclohexanol as CS+. After being conditioned, the flies were directly removed from the training system and put back into vials tubes containing wet filter paper until the reactivation phase.

Reactivation. Reactivation was performed $24 \mathrm{~h}$ after conditioning. This phase consisted in successive cycles of presentation of the CS+ alone without mechanics shocks. Each cycle consisted of a 1 min presentation of the CS+ followed by a $1 \mathrm{~min}$ rest (without odor). The resting period prevented habituation of the flies to the odorant. Flies received zero, one, three or five reactivation cycles and were then transferred back into vials containing wet filter paper until the memory assay.

Memory assay. A memory assay was performed either $24 \mathrm{~h}$ after initial conditioning or $24 \mathrm{~h}$ after reactivation. The test consisted of a choice between the CS + and the CS - . Each group was transferred to the central point of a T-maze, in which they were exposed to two convergent currents of air (one carrying CS+ and one carrying CS - ) from opposite arms of the T-maze. Flies were allowed to choose between the two odors for $30 \mathrm{~s}$, at which time they were trapped inside their respective arms of the T-maze, killed, and counted.

CXM treatment. To assess how consolidated memory depends on the synthesis of de novo proteins, flies were fed for $1 \mathrm{~h}$ with the protein synthesis inhibitor CXM (Sigma-Aldrich) dissolved into sweet water ( $20 \%$ sucrose) just before the conditioning or reactivation phase. Control flies were fed with sweet water only during the same amount of time. We used a dose $(35 \mathrm{~mm}$ ) previously shown to efficiently block LTM (Tully et al., 1994, Mery and Kawecki, 2002).

Statistical analysis. For each measurement, a memory score was calculated as the difference in the proportion of flies choosing octanol when conditioned to avoid methylcyclohexanol versus when conditioned to avoid octanol. For statistical comparison of the memory scores, all pro-

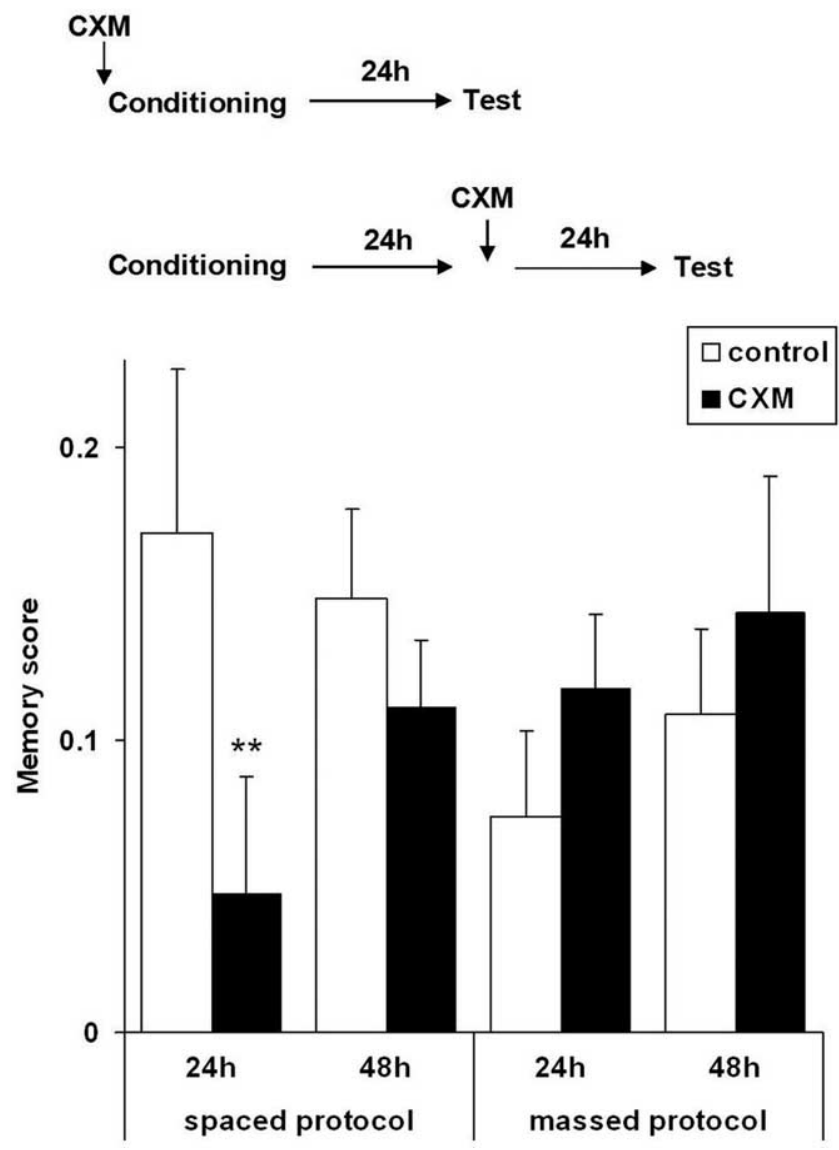

Figure 1. CXM sensitivity following a massed or spaced training acquisition protocol. Spaced protocol: memory was tested $24 \mathrm{~h}$ after conditioning (CXM just before conditioning: $n=44$ per group) or $48 \mathrm{~h}$ after conditioning (CXM $24 \mathrm{~h}$ after conditioning: $n=36$ per group). Massed protocol: memory was tested $24 \mathrm{~h}$ after conditioning ( $n=16$ per group) or $48 \mathrm{~h}$ after conditioning ( $n=16$ per group) $\left({ }^{* *} p<0.01\right)$. Error bars show SEM.

portions were angularly transformed before the analysis (Sokal and Rohlf, 1995). Differences between memory scores were tested with a univariate ANOVA. For comparisons among reactivation cycle number, we used planned orthogonal contrasts and $t$ tests were used for mean comparisons. All statistical analyses were performed using SPSS. $t$ tests were used on memory scores to evaluate whether they were significantly different to 0 .

\section{Results}

Massed and spaced conditioning induce stable ARM and LTM As a first step, we verified whether, using our protocol with a mechanical shock, we could obtain a CXM-resistant memory (ARM) after massed training and if spaced training also induced a CXM-sensitive memory (LTM). In our conditions, both massed and spaced training protocols of associative conditioning induced stable memory, as observed $24 \mathrm{~h}$ and $48 \mathrm{~h}$ later (Fig. 1). Flies treated with CXM before conditioning showed significantly reduced response levels compared with controls when the spaced protocol was used for conditioning (univariate ANOVA: $F_{(1,14)}=$ $10.3 ; p<0.01)$ but not with the massed protocol $\left(F_{(1,14)}=0.2\right.$; $p>0.05)$, thus indicating that LTM was formed only in the former case. However, once LTM was established, it became resistant to the blockade of protein synthesis: when applied $24 \mathrm{~h}$ after spaced conditioning, the CXM treatment did not affect response levels when tested $48 \mathrm{~h}$ postconditioning $\left(F_{(1,70)}=0.9\right.$; $p>0.05)$. We conclude that, in our experimental conditions, (1) the two protocols induce different stable memories (both ARM 

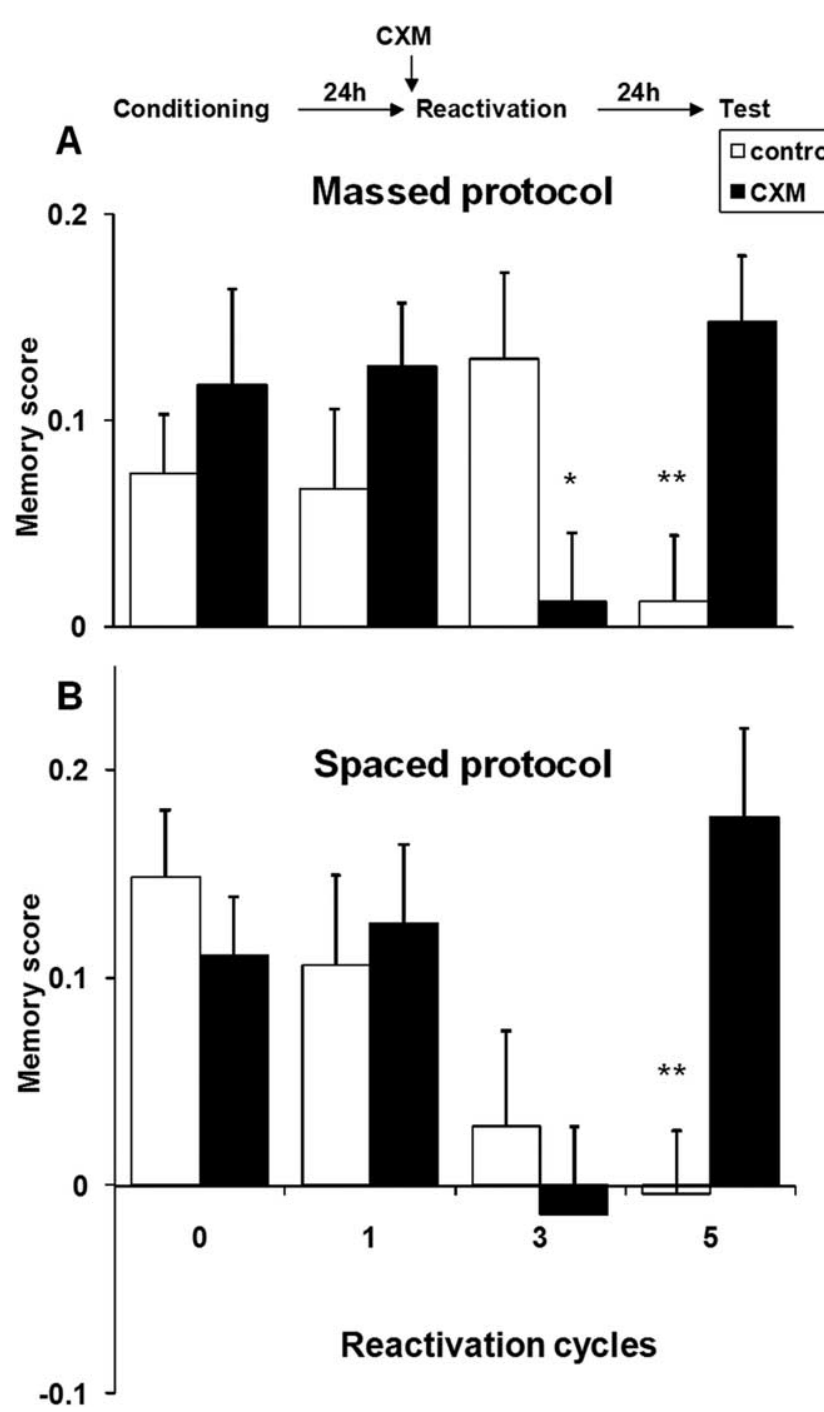

Figure 2. Effect of reactivation on stability of consolidated memory after a massed $(\boldsymbol{A})$ or a spaced $(\boldsymbol{B})$ protocol. $\boldsymbol{A}$, Zero, one, three, or five cycles of $\mathrm{CS}+$ re-exposure after massed conditioning (control group: $n=16$ per group; (XM-treated group: $n=15-16$ per group) $\boldsymbol{B}$, Zero, one, three or five cycles of $\mathrm{CS}+$ re-exposure $24 \mathrm{~h}$ after spaced conditioning (control group: 0 cycle, $n=36 ; 1$ cycle, $n=12 ; 3$ cycles, $n=12 ; 5$ cycles, $n=20$; CXM-treated group: 0 cycle, $n=36 ; 1$ cycle, $n=12 ; 3$ cycles, $n=12 ; 5$ cycles, $n=19)\left({ }^{*} p<0.05 ;{ }^{* *} p<0.01\right)$. Error bars Show SEM.

and LTM after spaced training and only ARM after massed training) and, (2) as expected, protein synthesis was required only for LTM formation but not for its maintenance nor for ARM formation.

\section{Moderate reactivation of ARM triggers a protein synthesis-dependent reconsolidation}

We then investigated the effect of the number of reactivation cycles on both stabilized memories. Twenty-four hours after a massed or spaced conditioning protocol, a variable number of reactivation cycles (see Materials and Methods) were performed, and the response levels of the flies were measured in a memory test $24 \mathrm{~h}$ later (i.e., $48 \mathrm{~h}$ after conditioning) (Fig. 2). One reactivation cycle did not affect memory, as shown by response levels similar to those of flies not submitted to any reactivation ( 0 cycle groups), whatever the conditioning protocol (planned contrast: LTM, $p>0.1$; ARM, $p>0.1$ ). Besides, whether they had been treated with CXM before the presentation of CS + had no effect
(Fig. 2) (one reactivation cycle, $p>0.1$ in both cases), thus confirming previous studies showing that a short reactivation does not affect initial consolidated memory.

When reactivation consisted of three cycles, the response levels observed $24 \mathrm{~h}$ later differed significantly according to the protocol used for conditioning. After a massed protocol, untreated flies submitted to a three-cycle reactivation responded at levels similar to those of non-reactivated flies (Fig. 2A). However, memory was abolished if flies had been treated with CXM before reactivation. This suggests that the initial memory trace was destabilized by the three-cycle reactivation and then consolidated through new protein synthesis. This is the first evidence, to our knowledge, of reconsolidation in Drosophila. Interestingly, the memory formed by the massed protocol conditioning was CXMresistant (Fig. 1), but it became CXM-sensitive after a three-cycle reactivation. Thus, it appears here that different mechanisms of stabilization were triggered after acquisition and after reactivation.

When tested $24 \mathrm{~h}$ after a spaced protocol conditioning and three reactivation cycles, neither CXM-treated nor control flies showed any significant memory (Fig. $2 \mathrm{~B}$ ). As this suggested that in such conditions the memory was less stable, we measured responses to the CS+ at shorter delays $(0,5$, or $8 \mathrm{~h}$ after reactivation) to test whether a memory trace could be detected at earlier times. However, surprisingly, we observed no significant memory at any of these time points (supplemental Fig. S1, available at www.jneurosci.org as supplemental material). From this, we conclude that, under our experimental conditions, when the initial memory is induced by spaced protocol (CXM-sensitive), three cycles of reactivation must disrupt it completely. This result is contradictory to previous studies which found a strong reconsolidation effect after reactivation on protein synthesis dependent acquisition memory (Nader et al., 2000).

Intense reactivation of both ARM and LTM produces protein synthesis-dependent extinction

When the number of reactivation cycles was increased to five, memory of the CS-US the response level of untreated flies was not significantly different from 0 , regardless of the protocol used to form the acquisition memory ( $t$ test: $p>0.1$ in both cases) (Fig. 2 ). Thus, the memory of the initial association was completely abolished $24 \mathrm{~h}$ later, and this effect was dependent on protein synthesis in both cases, since CXM-treated flies showed response levels similar to those of un-reactivated flies (planned contrast: $p>0.1$ in both cases). Thus, extinction induced by a five-cycle reactivation resulted in the active consolidation of a new memory, CS-noUS, expressed by a drastic drop of the response levels to the CS+. It is noteworthy that the requirement for protein synthesis in the stabilization of this new memory occurred independently of the involvement of protein synthesis in the consolidation of the acquisition memory, since similar results were obtained when the acquisition memory was CXM-resistant (Fig. $2 A$ ) and CXM sensitive (Fig. 2B).

Reconsolidation and extinction are specific to presentation of the CS+

Finally, we tested whether the observed reconsolidation and extinction processes were specific to the presentation of the CS+, by presenting the $\mathrm{CS}-$ instead during the reactivation cycles. As opposed to three cycles of CS + presentation, which induced significant CXM-sensitive reconsolidation of ARM (Fig. 3) $\left(\right.$ ANOVA: $\left.F_{(1,15)}=9.35, p<0.01\right)$, three cycles of CS - presentation $24 \mathrm{~h}$ after massed conditioning did not affect the response 

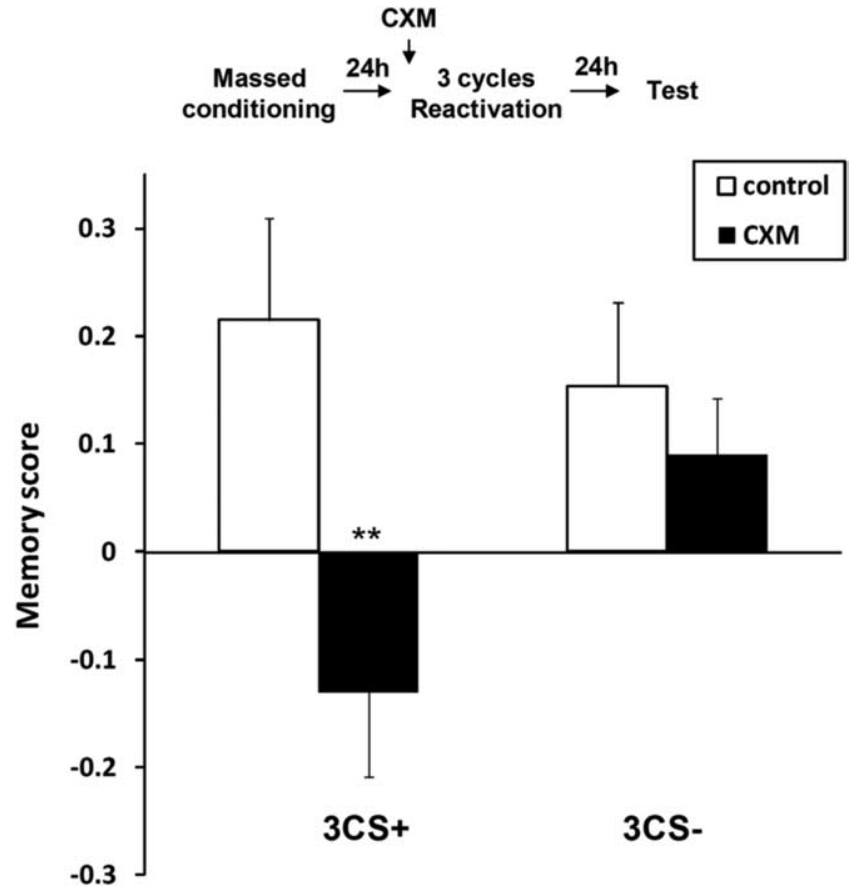

Figure 3. Specificity of moderate reactivation. Effect of $3 \mathrm{CS}+$ or $C S-$ exposure $24 \mathrm{~h}$ after a massed conditioning (ARM). CXM treatment is administrated just before reactivation (3CS+: $n=8$ per group; $3 C S-: n=8$ per group) $\left.{ }^{* *} p<0.01\right)$. Error bars show SEM.

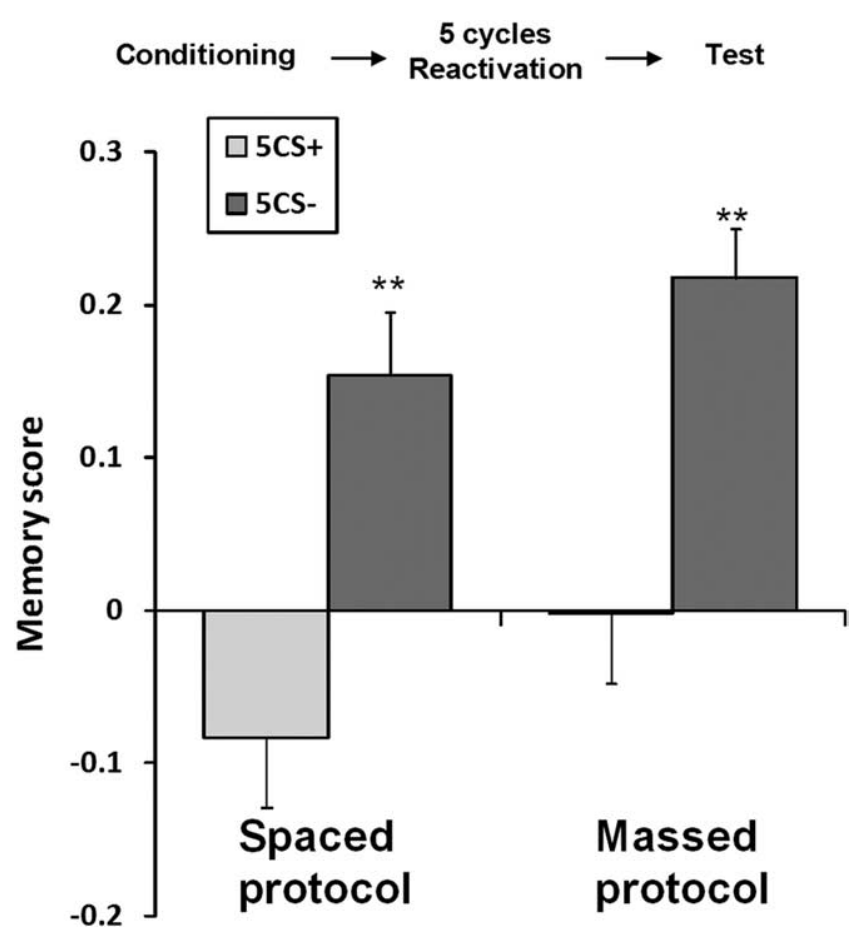

Figure 4. Specificity of intense reactivation. Effect of $5 \mathrm{CS}+$ or $\mathrm{CS}-$ exposure $24 \mathrm{~h}$ after spaced ( $n=8$ per group) or massed conditioning ( $n=8$ per group) $\left({ }^{* *} p<0.01\right)$. Error bars ShOW SEM.

of the flies whether they had been treated with CXM or not (Fig. 3) $\left(F_{(1,14)}=0.16, p>0.05\right)$. Similarly, while extinction occurred after a $5 \mathrm{CS}+$ reactivation cycle, i.e., flies showed no significant memory ( $t$ test: ARM: $t_{(1,7)}=-1.9, p>0.05$; LTM: $t_{(1,7)}=$ $-0.035, p>0.05)$, flies exposed to $5 \mathrm{CS}-$ reactivation cycles still responded significantly to the $\mathrm{CS}+\left(\mathrm{ARM}: t_{(1,7)}=4.55, p<0.01\right.$;
LTM: $t_{(1,7)}=4.22, p<0.01$ ) (Fig. 4). Thus, extinction of both ARM and LTM could be induced by a sufficient number of presentations of the CS+ only. We therefore conclude that, when occurring, reconsolidation and extinction were specifically triggered by repeated presentations of the CS+.

\section{Discussion}

In this study, we addressed for the first time the question of the relationship between consolidation, reconsolidation and extinction using Drosophila melanogaster. Our results clearly show that both reconsolidation and extinction exist in this model. This opens new perspectives for the understanding of the molecular and cellular aspects of such processes. The wide array of genetic tools now available in this model organism can be used to tackle these questions, as it allowed dissecting several aspects of memory stabilization (Keene and Waddell, 2007). Within this perspective, we have started investigating the relationships between extinction, reconsolidation and stabilization of acquisition memory. For this, we took advantage of a potentially unique aspect of Drosophila cognition: the existence of two forms of stabilized memory, ARM and LTM, respectively resistant and sensitive to the CXM protein synthesis inhibitor (Margulies et al., 2005). We asked whether this dichotomy extended to reconsolidation and extinction. Our findings show that (1) whether reconsolidation or extinction takes place upon reactivation of the stable memory depends on the intensity of such reactivation, (2) both processes can be built whether the memory initially stabilized was CXMsensitive or resistant, (3) both are CXM-sensitive even if the stabilization of the acquisition memory was not, as in the case of ARM, and (4) extinction or reconsolidation occurs specifically when reactivation involves the presentation of the CS+.

\section{The onset of reconsolidation or extinction depends on the intensity of reactivation by $\mathrm{CS}+$ presentations}

Together, our findings show that after formation of an acquisition memory through repeated CS-US pairings, further reactivation of this memory can modify the flies' response to the CS. The observed change is variable, depending on the intensity of reactivation (here, the number of reactivation cycles). This is in accordance with previous studies on other organisms (crab: Pedreira and Maldonado, 2003; fish: Eisenberg and Dudai, 2004; rat: Suzuki et al., 2004; Power et al., 2006; bee: Stollhoff et al., 2005). Here, while a weak reactivation (one cycle) did not modify the expression of the acquisition memory (CS-US), a moderate (three cycles) or intense (five cycles) reactivation could induce, respectively, reconsolidation of the acquisition memory or extinction. The latter is generally considered to result from the formation of a new memory (extinction memory) corresponding to the new association of the CS with the absence of US. This view is comforted by our demonstration that the CS+ is required to induce the observed drop of conditioned responses (CS - presentations had no effect). Besides, extinction is an active process involving protein synthesis, since a CXM treatment maintains stable levels of response after five reactivations. We interpret this as the expression of the original acquisition memory when the formation of the new extinction memory is repressed, in accordance with previous work (Suzuki et al., 2004; Stollhoff et al., 2005). Thus, in Drosophila as well, extinction corresponds to the consolidation of a new memory trace that is sensitive to protein synthesis inhibitors rather than to the erasure of the initial trace.

If extinction is not induced, the original memory can be maintained over days once formed and stabilized, and is resistant to CXM treatment, independently of the conditioning protocol. 
However, we observed reduced response levels in CXM-treated flies after three cycles of reactivation (after a massed conditioning). This result thus indicates that moderate reactivation can destabilize the originally CXM-resistant memory and turn it into a labile, CXM-sensitive, memory. This effect of moderate reactivation has been observed in a wide range of studies and has been interpreted as a reconsolidation process (for review, see Tronson and Taylor, 2007). However, in this study, it is surprising that we could not reveal such a reconsolidation after spaced training acquisition, since both CXM-treated and untreated flies maintained low levels of response when identical reactivation conditions followed spaced conditioning. Although reconsolidation should lead to a stable memory, we tested the unlikely possibility that memory had been reconsolidated indeed but then had been quickly erased during the $24 \mathrm{~h}$ delay before testing. Our results clearly show that no expression of memory could be observed, even immediately after reactivation. This is at odds with previous studies which found a strong reconsolidation effect after reactivation of protein synthesis-dependent acquisition memory (Nader et al., 2000). It is neither likely that extinction occurred, since this drop in expression of the CS-US memory was observed also in CXM-treated flies. Indeed, CXM should have repressed extinction memory and allowed normal response levels, like after five cycles of reactivation (see above), though we cannot discard that some CXM-resistant extinction might have occurred. Neither can we exclude the fact that under these conditions reconsolidation and extinction might have co-occurred. This remains an open question that will require further studies. Still, our main result remains that both extinction and reconsolidation could be demonstrated experimentally in Drosophila. As in other species, these opposite processes share a common requirement for protein synthesis, and are triggered by different intensities of reactivation (Eisenhardt and Menzel, 2007).

\section{Reconsolidation does not recapitulate consolidation in Drosophila}

Interestingly, bona fide reconsolidation, depending on protein synthesis, could be observed after reactivation of acquisition memory of the ARM type, which had been stabilized independently of protein synthesis. If reconsolidation was underlined by mechanisms identical to those of consolidation, we would expect two possible outcomes. If one considers that ARM stabilization does not require protein synthesis and thus is not proper consolidation, reconsolidation should not occur after ARM formation. Alternatively, considering that ARM was consolidated in a CXMresistant manner (if consolidation is taken sensu lato as stabilization), reconsolidation should occur likewise. However, our results show that, in flies submitted to a massed conditioning followed by three cycles of reactivation, two different events occurred: first, an ARM-type acquisition memory was formed and stabilized (through CXM-resistant mechanisms yet unknown); second, this memory was reactivated and destabilized, then reconsolidated into a CXM-sensitive form of memory. Even if considering that CXM does not fully block protein synthesis (Tully et al., 1994), it remains clear that we are in presence of two different forms of memory, and that the stabilization of the CXM-sensitive reconsolidated memory must involve different processes from those contributing to the formation of the original CXMresistant acquisition memory. This conclusion confirms the view that reconsolidation is not a repetition of consolidation (Nader et al., 2000; Alberini, 2005), and extends it to Drosophila, a new model thus available now for the study of these questions.

\section{A molecular switch triggered by reactivation?}

In our experimental conditions, acquisition could lead to two different forms of memory depending whether massed (ARM) or spaced trials (LTM and ARM) were used during conditioning, in accordance with previous studies (Tully et al., 1994). On the contrary, it is remarkable that, as far as we have observed, postreactivation processes appear more unified as they required protein synthesis in all our experiments. In particular, both massed and spaced conditioning could be followed by the formation of an extinction memory which was disrupted by CXM, regardless of the nature of the initial memory (LTM and/or ARM). More specifically, it is striking that an ARM-type memory is not sensitive to the protein synthesis inhibitor for its stabilization, but that its extinction and reconsolidation are. Thus, as proposed above for reconsolidation, we conclude that molecular pathways involving protein synthesis could be recruited for extinction even if they had not been required initially for the formation of the acquisition memory. Hence, extinction and acquisition memories can be stabilized through distinct underlying mechanisms. Interestingly, we used a reactivation protocol in which the CS+ presentations followed each other without delay. This can thus be viewed as a massed conditioning protocol for the acquisition of the CS-noUS memory (extinction memory). The rule that massed training leads to CXM-resistant memory would then not apply to extinction, for which a CXM-sensitive memory trace does not necessarily require spaced trials to be established. We can therefore conclude that post-reactivation processes like extinction and reconsolidation involve protein synthesis in such a way that they can be disrupted by CXM, even if they follow the stabilization of ARM (CXM-resistant), at least under our experimental conditions. This implies that a switch between different signaling pathways may take place upon reactivation, as those involved initially in consolidation may not be used. Perazzona et al. (2004) have proposed that antagonistic molecular cascades (cAMP-dependent for LTM and cAMP-independent for ARM) could underlie the formation of ARM and LTM after acquisition. According to their model, ARM would serve as a gating mechanism for LTM formation. These authors showed that if LTM was formed, it implied that ARM should be erased. Our results suggest that this may also apply to LTM-type extinction and reconsolidation memories. This competition is still under debate, and whether it is symmetrical remains an open question, as inhibition of LTM by ARM has only been hypothesized (Isabel et al., 2004). Interestingly, we find that destabilization of ARM can be followed by consolidation of reconsolidation memory through protein synthesis. Future work with mutants should help to test the implication of cAMP-dependent and independent pathways in post-reactivation processes.

\section{References}

Alberini CM (2005) Mechanisms of memory stabilization: are consolidation and reconsolidation similar or distinct process? Trends Neurosci 28:51-56

Alberini CM, Milekic MH, Tronel S (2006) Mechanisms of memory stabilization and de-stabilization. Cell Mol Life Sci 63:999-1008.

Dudai Y (2006) Reconsolidation: the advantage of being refocused. Curr Op Neurobiol 16:174-178.

Dudai Y, Eisenberg M (2004) Rites of passage of the engram: reconsolidation and the lingering consolidation hypothesis. Neuron 44:93-100.

Dudai Y, Morris R (2000) To consolidate or not to consolidate: what are the questions? In: Brain, perception, memory. Advances in cognitive sciences (Bolhius J, ed), pp 149-162. Oxford: Oxford UP.

Duncan CP (1949) The retroactive effect of electroconvulsive shock. J Comp Physiol Psychol 42:32-44.

Eisenberg M, Dudai Y (2004) Reconsolidation of fresh, remote and extin- 
guished fear memory in medaka: old fears don't die. Eur J Neurosci 20:3397-3403.

Eisenhardt D, Menzel R (2007) Extinction learning, reconsolidation and the internal reinforcement hypothesis. Neurobiol Learn Mem 87:167-173.

Isabel G, Pascual A, Preat T (2004) Exclusive consolidated memory phases in Drosophila. Science 304:1024-1027.

Keene AC, Waddell S (2007) Drosophila olfactory memory: single genes to complex neural circuits. Nat Rev Neurosci 8:341-354.

Mactutus CF, Riccio DC, Ferek JM (1979) Retrograde amnesia for old (reactivated) memory: some anomalous characteristics. Science 204:1319-1320.

Margulies C, Tully T, Dubnau J (2005) Deconstructing memory in Drosophila. Cur Biol 15:R700-R713.

McGaugh JL (2000) Memory - a century of consolidation. Science 287:248-251.

Mery F, Kawecki TJ (2002) Experimental evolution of memory ability in fruit flies. Proc Natl Acad Sci U S A 99:14274-14279.

Mery F, Kawecki TJ (2005) A cost of long-term memory in Drosophila. Science 308:1148.

Misanin JR, Miller RR, Lewis DJ (1968) Retrograde amnesia produced by electroconvulsive shock after reactivation of consolidated memory trace. Science 160:554-555.

Moore JL, Roche RAP (2007) Reconsolidation revisited: a review and commentary on the phenomenon. Rev Neurosci 18:365-382.

Nader K, Schafe GE, Le Doux JE (2000) Fear memories require protein synthesis in the amygdale for reconsolidation after retrieval. Nature 406:722-726.
Nader K, Hardt O, Wang SH (2005) Response to Alberini: right answer, wrong question. Trends Neurosci 28:346-347.

Pedreira ME, Maldonado H (2003) Protein synthesis subserves reconsolidation or extinction depending on reminder duration. Neuron 38:863-869.

Perazzona B, Isabel G, Preat T, Davis RL (2004) The role of cAMP response element-binding protein in Drosophila long-term memory. J Neurosci 24:8823-8828.

Power AE, Berlau DJ, McGaugh JL, Steward O (2006) Anisomycin infused into the hippocampus fails to block "reconsolidation" but impairs extinction. The role of re-exposure duration. Learn Mem 13:27-34.

Sara SJ (2000) Retrieval and reconsolidation: toward a neurobiology of remembering. Learn Mem 7:73-84.

Sokal RR, Rohlf FJ (1995) Biometry: the principles and practice of statistics in biological research, Ed 3. New York: W. H. Freeman.

Stollhoff N, Menzel R, Eisenhardt D (2005) Spontaneous recovery from extinction depends on the reconsolidation of the acquisition memory in an appetitive learning in the honeybee Apis mellifera. J Neurosci 25:4485-4492.

Stollhoff N, Menzel R, Eisenhardt D (2008) One retrieved trial induces reconsolidation in an appetitive learning paradigm in honeybees (Apis mellifera). Neurobiol Learn Mem 89:419-425.

Suzuki A, Josselyn SA, Frankland PW, Masushige S, Silva AJ, Kida S (2004) Memory reconsolidation and extinction have distinct temporal and biochemical signatures. J Neurosci 24:4787-4795.

Tronson NC, Taylor JR (2007) Molecular mechanisms of memory reconsolidation. Nat Rev Neurosci 8:262-275.

Tully T, Preat T, Boynton SC, Del Vecchio M (1994) Genetic dissection of consolidated memory in Drosophila. Cell 79:35-47. 